\title{
Histopathological changes in myocardial tissue due to coronary venous hypertension
}

\author{
Ercan Akşit ${ }^{1}$, Başak Büyük² ${ }^{2}$ Sonay Oğuz ${ }^{3}$
}

${ }^{1}$ Department of Cardiology, Faculty of Medicine, Canakkale Onsekiz Mart University, Canakkale, Turkey

2Department of Histology and Embryology, Faculty of Medicine, Canakkale Onsekiz Mart University, Canakkale, Turkey

${ }^{3}$ Department of Cardiovascular Surgery, Faculty of Medicine, Canakkale Onsekiz Mart University, Canakkale, Turkey

Submitted: 2 August 2019

Accepted: 1 December 2019

Arch Med Sci

DOI: https://doi.org/10.5114/aoms.2019.91472

Copyright $\odot 2019$ Termedia \& Banach

\author{
Corresponding author: \\ Asst. Prof. Ercan Akşit \\ Department of Cardiology \\ Faculty of Medicine \\ Onsekiz Mart University \\ Barbaros Street Terzioglu \\ Campus B Block No. 4 \\ Canakkale, Turkey \\ Phone: +902862635950 \\ Fax: +902862635956 \\ E-mail: ercanaksit@comu. \\ edu.tr
}

\begin{abstract}
Introduction: In chronic venous insufficiency (CVI), an increase in venous pressure causes the passage of intravascular blood cells and molecules into the surrounding tissues and induces histopathological changes in the lower extremities, leading to increased pigmentation in the legs, ulceration, and tissue loss to various degrees. This study aimed to investigate whether an increase in venous pressure in the coronary veins can lead to the aforementioned histopathological changes.

Material and methods: Twenty-four New Zealand rabbits were divided into the following three groups: experimental model of coronary venous hypertension (CVH) $(n=8)$, sham group $(n=8)$, and control group $(n=8)$. After 21 days postoperatively, tissue samples from each group were compared for perivascular inflammation, erythrocyte extravasation, macrophage infiltration, and hemosiderin deposits by histopathological scoring under a light microscope. Matrix metalloproteinase-2 (MMP-2) activation was evaluated using immunohistochemical staining.

Results: In the CVH group, hemosiderin accumulation was significantly higher than in the sham and control groups (1.0 (1.0-3.0), $0.0(0.0-1.0), 0.0(0.0-$ $0.0) ; p<0.001)$. Immunohistochemically, in the CVH group, MMP-2 levels were significantly higher than in the sham and control groups $(2.0$ (1.0-3.0), 0.0 (0.0-1.0), $0.0(0.0-0.0) ; p<0.001)$.

Conclusions: This experimental study showed for the first time the histopathological and immunohistochemical changes in myocardial tissue, similar to those observed in CVI, as a result of increased coronary venous pressure due to coronary vein ligation. Further studies are needed to understand the clinical implications of these results.
\end{abstract}

Key words: coronary veins, hemosiderin, Thebesian valve, vein occlusion, venous insufficiency.

\section{Introduction}

Cardiovascular system diseases are still the leading cause of morbidity and mortality globally [1]. Chronic venous insufficiency (CVI) is a clinical condition observed in the lower extremities as a result of venous hypertension caused by venous valve insufficiency, venous flow obstruction or both. Although the most common cause is increased 
venous pressure due to valve insufficiency, CVI is the most commonly used term for all etiologic and clinical manifestations of chronic venous disease [2]. This pressure load on the lower extremity veins causes blood cells and molecules in the veins to pass into the surrounding tissue, leading to tissue damage. Erythrocytes that exit the vein due to increased intravenous pressure, resulting from venous stasis, are degraded by interstitial macrophages, causing iron accumulation in the tissue. Eventually, the increased iron load causes ferrite to undergo a structural change, converting it to hemosiderin. Iron overload and other metabolites accumulating disrupt tissue structure and cause tissue damage to various degrees $[3,4]$. This histopathological phenomenon results in venous leg ulcers in some patients. In addition to standard medical and surgical treatment for these venous leg ulcers, autogenously derived regenerative cell therapy is also applied in selected cases [5].

Valves are widely found in coronary veins. Thebesian valves are located in the coronary sinus (CS) ostium. Vieussens valves are also observed at the point where the great cardiac vein intersects the posterolateral vein. Valves are mostly located in the great cardiac vein, inferior interventricular vein, and posterior cardiac vein of the left ventricle, with an average of 2.5-3 valves per vein [6]. A study demonstrated that valves in the coronary veins were functional and prevented retrograde flow against gravity [7]. Thus, we hypothesized that an increase in the coronary venous pressure can have histopathological outcomes in the myocardial tissue. For this purpose, we increased intravenous pressure in the coronary vein using a coronary vein ligation model and investigated whether the histopathological changes observed in the lower extremity tissue during CVI were also observed in the myocardial tissue.

\section{Material and methods}

\section{Experimental animals}

The study was approved by the Institutional Animal Use and Care Committee of Canakkale Onsekiz Mart University and was conducted in accordance with the Helsinki Declaration of World Medical Association recommendations on animal studies (Protocol no: 2018/10-08). Twenty-four male New Zealand rabbits (age: 11-14 months) weighing $2.2-3.4 \mathrm{~kg}$ were used in this study. Standard rabbit feed and water were provided throughout the study. The rabbits were individually housed in a special steel cage in a special room at $22 \pm 2^{\circ} \mathrm{C}$ and $55-60 \%$ humidity with a $12-\mathrm{h}$ dark/light cycle.

\section{Study groups}

The rabbits were randomized into the following three groups:

1. $\mathrm{CVH}$ group $(n=8)$ : left thoracotomy was performed on the rabbits to access the coronary veins, which were ligated.

2. Sham group (pseudo-surgery; $n=8$ ): the rabbits underwent left thoracotomy without coronary vein ligation.

3. Control group ( $n=8)$ : no surgical procedure was performed in this group, and tissue samples were obtained after the rabbits were sacrificed at the same time with the other two groups.

Reportedly, histopathological and immunohistochemical changes were observed in the lower extremity tissues on the $21^{\text {st }}$ day in CVI experimental models created using femoral vein ligation [8]. Thus, in the present study, myocardial tissue samples were obtained from all animals after 21 postoperative days.

\section{Anesthesia protocol}

Rabbits were intramuscularly administered xylazine $(10 \mathrm{mg} / \mathrm{kg})$ and ketamine hydrochloride (40 mg/kg).

\section{Coronary vein ligation model}

For planning our experimental model, experimental models of defined venous insufficiency of other systems and organs were examined in detail. It was observed that femoral vein ligation [8, 9] has been performed to study CVI, jugular vein ligation [10] to study chronic cerebrospinal venous insufficiency (CCSVI) and ovarian vein ligation [11] to study ovarian venous insufficiency. Thus, coronary vein ligation was planned to test our hypothesis. Our aim was to find an appropriate location that was far away from the distal vein, where all coronary veins drain, but that technically allowed ligation. The rabbit coronary vein anatomy was evaluated carefully before the procedure. Rabbits do not have a CS; instead, the great cardiac vein (GCV), which is the largest coronary vein in rabbits, opens directly into the left cranial vena cava (LCVC). The GCV comprises two main parts: one is the left circumflex vein (LCX) and the other is the paraconal interventricular vein (PIV), which takes course in the paraconal interventricular groove and ends near the apex cordis. The PIV gives collateral branches, with distal branches extending to the right and left ventricles [12]. The GCV and LCX cannot be seen in a cranial view, as they remain hidden under the left auricle [12, 13] (Figure 1). In the light of this information, the surgical procedure was initiated.

Cefazolin sodium (intramuscular; $50 \mathrm{mg} / \mathrm{kg}$ ) was preoperatively administered to prevent in- 
fection. The surgical procedure was initiated after the primary practitioner established a view with a $3.5 \times$ magnification surgical loupe. Subcutaneous muscle tissue was accessed by incising the skin from the anterolateral region corresponding to the third intercostal space. The lower part of the third intercostal space was opened by blunt dissection to prevent damage to the left hemithorax. An intercostal width of approximately $2.5-3 \mathrm{~cm}$ was achieved. Using a mini retractor, the left hemithorax was accessed after rib retraction. After the pericardium and the heart were visualized in the medial area, the pericardial space was accessed by opening the pericardium from the inferioposterior line to the superioanterior line. As myocardial infarction, shock, and sudden death due to CS thrombosis have been reported in experimental models in dogs and case reports, tying the vein in a region distant from the GCV was preferred [14, 15]. At the paraconal interventricular groove region, an epicardial 7/0 prolene suture was precisely passed from the distal side of the point where the PIV drained into the GCV. To ensure that only the PIV was ligated, suturing was performed before the ligation was taken to traction; moreover, it was checked whether the distal vein filled and stretched (Figure 1). After $30 \mathrm{~s}$, assessment was performed for determining coronary ischemia findings. After ensuring that the coronary artery was not included in the suture line, venous passage was blocked by ligating the vein segment. At the end of the 21 days, rabbits were operated on using the same surgical procedure under general anesthesia to reach the heart, and euthanasia was performed by exsanguination [16]. The heart tissue was fully excised. Ligation sites were identified with the aid of sutures.

Because the rabbit heart is very small and one cannot predict how far the damage spreads in this model of coronary vein hypertension, CVI and CCSVI experimental models were referenced for histopathological sampling $[9,10]$. In these two models, tissue samples taken from tissues under the ligated vein segment were compared with those taken from the sham and control groups. Myocardial tissue in the $1 \mathrm{~cm}^{2}$ area under coronary vein ligation was enumerated by excising the tissue that corresponded to the same region in the sham and control groups.

\section{Histopathological evaluation}

Histopathological evaluation was performed in the histology department of Canakkale Onsekiz Mart University by an expert histologist who was blinded to the groups from which tissues were obtained. The tissues were immediately fixed in $10 \%$ neutral buffered formalin for $48 \mathrm{~h}$. After fixation, dehydration, pellucidation, and paraffin

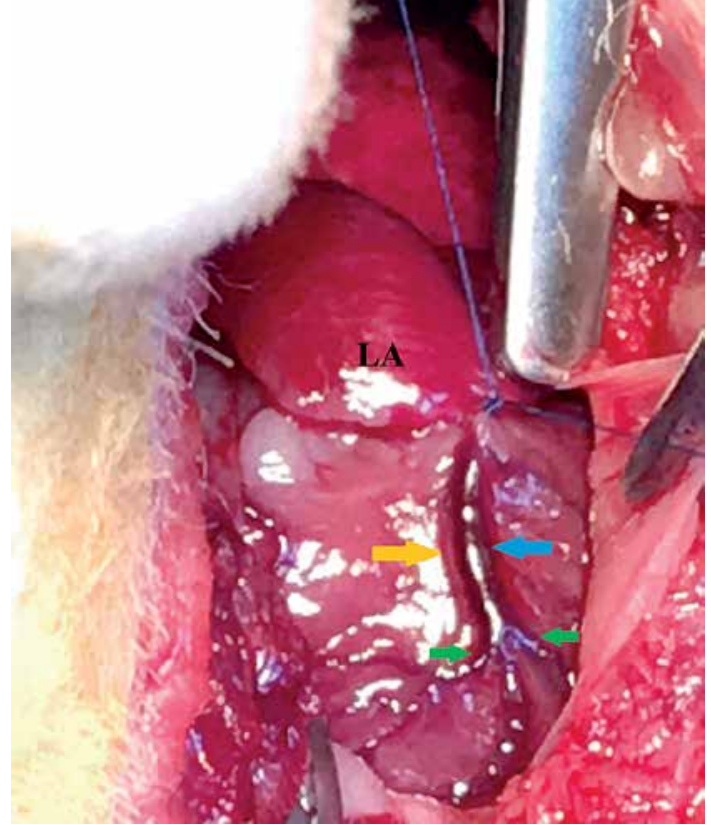

Figure 1. Cranial surface of the New Zealand rabbit heart. The paraconal interventricular vein (blue arrow) has swollen after ligation. The paraconal interventricular artery (yellow arrow). The collateral veins (distal branches of paraconal interventricular veins) (green arrow)

LA - left auricle.

embedding, 4-5- $\mu$ l sections were obtained from the paraffin blocks using a Rotary Microtome (Leica RM2125 RTS). Samples were evaluated under a light microscope (Zeiss AxioScope A1). Perivascular inflammation, erythrocyte extravasation, and macrophage infiltration were graded using routine hematoxylin and eosin staining. Hemosiderin deposits in the tissue were graded using Prussian blue staining. Scoring for histopathological grading was as follows: 0 , no findings in the area examined using the light microscope; 1 - findings noted in $<25 \%$ of the examined area; 2 - findings noted in $25 \%-75 \%$ of the examined area; and 3 - findings in $>75 \%$ of the examined area [17].

\section{Immunohistochemical staining}

Immunohistochemical staining was performed using the matrix metalloproteinase-2 (MMP-2) monoclonal antibody kit (lot no: 2776852, EMD Millipore corporation, USA) according to the manufacturer's instructions. Immunohistochemical scoring of MMP-2 was performed according to the staining intensity of the slides ( 0 - no staining; 1 - poor but detectable staining; 2 - moderate staining; and 3 - strong staining).

\section{Statistical analysis}

Data were analyzed using SPSS version 21.0. Descriptive data were presented as median (min- 
imum-maximum). The Kruskal-Wallis test was used to compare the variables. The Mann-Whitney $U$ test with Bonferroni correction was used for comparing data. A value of $p<0.05$ was considered as statistically significant.

\section{Results}

\section{Histopathologic findings}

In the CVH group, perivascular inflammation was significantly higher than in the sham and control groups (2.5 (1.0-3.0), 0.0 (0.0-1.0), 0.0 (0.0$0.0) ; p<0.001)$. In the $\mathrm{CVH}$ group, erythrocyte extravasation was significantly higher compared to the sham and control groups (3.0 (2.0-3.0), 0.5 (0.0-1.0), 0.0 (0.0-0.0); $p<0.001)$. The $\mathrm{CVH}$ group demonstrated significantly higher macrophage infiltration than the sham and control groups (3.0 (2.0-3.0), 0.5 (0.0-1.0), 0.0 (0.0-1.0); $p<0.001)$. The $\mathrm{CVH}$ group also demonstrated significantly higher hemosiderin accumulation than the sham and control groups (1.0 (1.0-3.0), 0.0
(0.0-1.0), $0.0(0.0-0.0) ; p<0.001)$. Figure 2 presents images of histopathological samples under light microscopy.

\section{Immunohistochemical findings}

Immunohistochemical evaluation revealed that MMP-2 levels in the myocardial tissue around the coronary vein were significantly higher in the $\mathrm{CVH}$ group than those in the sham and control groups (2.0 (1.0-3.0), 0.0 (0.0-1.0), 0.0 (0.0-0.0); $p<$ 0.001 ). Figure 3 presents images of immunohistochemical MMP-2 staining under light microscopy. Histopathological and immunohistochemical scores of the groups are shown in Table I.

\section{Discussion}

In this study, we demonstrated erythrocyte extravasation, macrophage infiltration, hemosiderin accumulation and MMP-2 staining in myocardial tissue as a result of increased coronary venous pressure. The clinical impact of histopathological
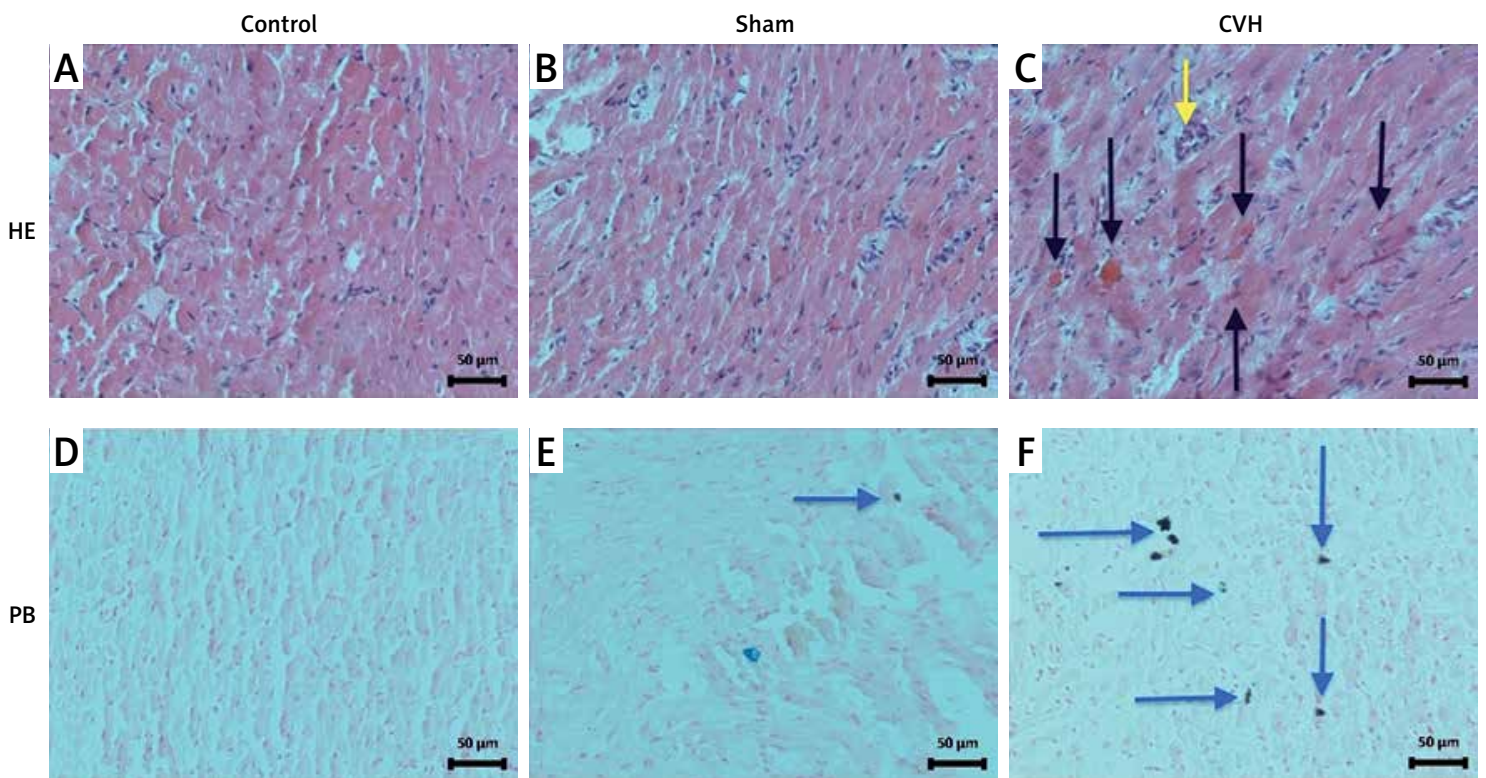

Figure 2. Hematoxylin-eosin (HE) stained (A, B, and C) and Prussian blue (PB) stained (D, E, and F) microscopic images of the control, sham, and coronary venous hypertension (CVH) groups, respectively. Erythrocyte extravasation seen in routine histopathological evaluation is indicated with black arrows, whereas macrophage infiltration is indicated with a yellow arrow. Hemosiderin deposits in PB staining are indicated with blue arrows (magnification, 200x)
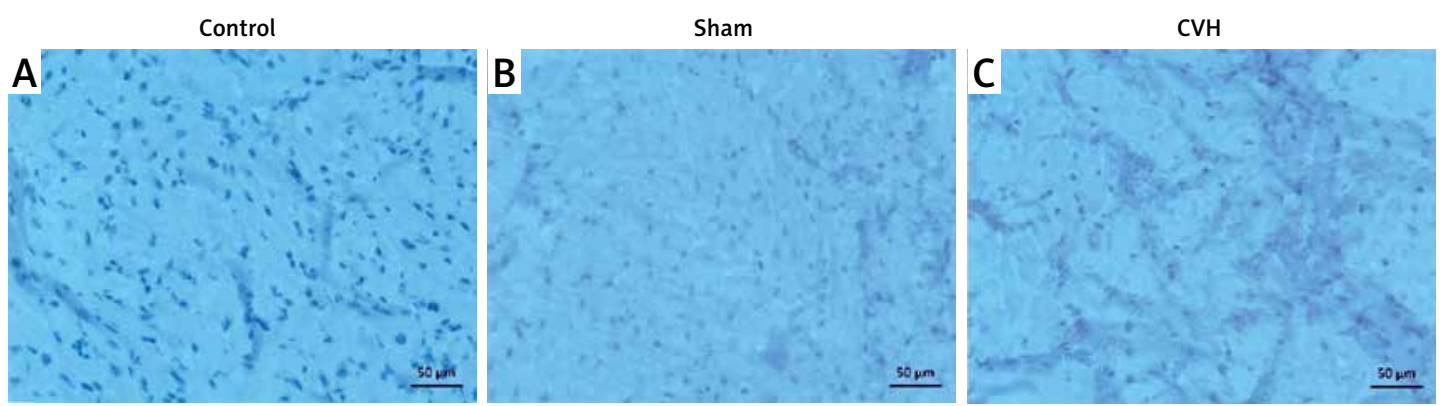

Figure 3. MMP-2 immunohistochemical staining of control (A), sham (B), and coronary venous hypertension (CVH)

(C) groups. MMP-2 staining increases from A to C (magnification, 200x) 
Table I. Histopathological and immunohistochemical scores of the groups

\begin{tabular}{|lccccc|}
\hline Group & $\begin{array}{c}\text { Perivascular } \\
\text { inflammation }\end{array}$ & $\begin{array}{c}\text { Erythrocyte } \\
\text { extravasation }\end{array}$ & $\begin{array}{c}\text { Macrophage } \\
\text { infiltration }\end{array}$ & $\begin{array}{c}\text { Hemosiderin } \\
\text { accumulation }\end{array}$ & MMP-2 staining \\
\hline Control & $0.0(0.0-0.0)$ & $0.0(0.0-0.0)$ & $0.0(0.0-1.0)$ & $0.0(0.0-0.0)$ & $0.0(0.0-0.0)$ \\
\hline Sham & $0.0(0.0-1.0)$ & $0.5(0.0-1.0)$ & $0.5(0.0-1.0)$ & $0.0(0.0-1.0)$ & $0.0(0.0-1.0)$ \\
\hline CVH & $2.5(1.0-3.0)$ & $3.0(2.0-3.0)$ & $3.0(2.0-3.0)$ & $1.0(1.0-3.0)$ & $2.0(1.0-3.0)$ \\
\hline$P$-value & $<0.001^{*}$ & $<0.001^{*}$ & $<0.001^{*}$ & $<0.001^{*}$ & $<0.001^{*}$ \\
\hline
\end{tabular}

Data are presented as median (minimum-maximum); ${ }^{*} p<0.05$. CVH-coronary venous hypertension, MMP-2-matrix metalloproteinase-2.

changes in the lower extremity during CVI is well defined by both experimental and clinical studies. In animal studies conducted for CVI, an arteriovenous fistula model was created to increase venous pressure, which demonstrated that an increased intravenous pressure led to venous hypertension, causing leukocyte infiltration and especially macrophage accumulation, in tissues approximately 21 days later [18]. Hahn et al. tied the femoral vein of rats and observed intense leukocyte infiltration in the skin of the lower extremities on day 7 [9]. In their study, Herrick et al. biopsied a region of the lower extremity with venous ulcer and demonstrated the presence of intense polymorphonuclear leukocytes, focal erythrocyte extravasation, and small, perivascular, dense hemosiderin deposits in this region [19]. High MMP-2 levels in lower extremity venous ulcers were also demonstrated in an earlier study [20]. In the present study, we found that perivascular inflammation, erythrocyte extravasation, and hemosiderin accumulation increased significantly in the CVH group compared with those in the control and sham groups.

Cardiac hemochromatosis is a disease that leads to excessive accumulation of iron in myocytes and may cause systolic and diastolic dysfunctions in the left ventricle. Reportedly, iron accumulation in these patients is responsible for many pathologies such as angina pectoris and pericardial constriction. Iron deposits accumulated in the myocytes may cause atrial and ventricular tachycardias with non-homogeneous electrical conduction and prolonged repolarization. Chronic iron overload reduces CaV1.3-dependent L-type Ca flows, leading to the development of bradycardia, electrical conduction, and atrial fibrillation. A reduction in left ventricular dilatation and ejection fraction may cause ventricular arrhythmia and sudden death [21]. A recent study revealed that myocardial fibrosis and hepatic fibrosis result from iron-mediated injury and that a significant increase in MMP-2 level is observed in both myocardial and liver tissues [22]. Furthermore, it is well known that serum MMP-2 levels increase in hemochromatosis and correlate with the severity of hepatic fibrosis [23]. In the present study, we also observed intense MMP-2 staining in the CVH group compared with that in the other groups. Hemosiderin deposits, whose presence we have histopathologically demonstrated in $\mathrm{CVH}$, may lead to hemochromatosis-like cardiomyopathy and cause other similar clinical manifestations observed in hemochromatosis. CCSVI is characterized by a number of stenotic malformations affecting the extracranial cerebrospinal venous outflow and especially the internal jugular and azygous veins. The observation of iron overload and pericapillary fibrin cuffs in the vein wall, which are an indicator of venous hypertension, in patients with multiple sclerosis (MS) has led to the hypothesis that CCSVI is a cause of MS [24]. Reportedly, iron overload directly damages the tissue by oxidative stress and indirectly by leading to the primary activation of the autoimmune cascade [25]. Zakaria et al. performed jugular vein ligation in rats and followed them for 8 months. In the jugular vein ligation group, intense iron accumulation was observed in the corpus callosum and cingulum and along the white matter tracts containing external capsules [10]. Similar to the dense iron accumulation in the white matter in CCSVI after jugular vein ligation, dense hemosiderin accumulation was observed in myocardial tissue in our experimental model.

There are many clinical conditions in which the volume of the coronary veins, especially that of the coronary sinus, is increased, leading to increased coronary venous pressure. Coronary arteriovenous fistulas may be congenital or acquired, and in these anomalies, some of the arteries may be drained into the CS [26]. Persistent left superior vena cava (PLSVC) is a condition in which the veins of the heart expand [27, 28]. Giant cardiac varices have been demonstrated in PLSVC with CS thrombosis [29]. Coronary venous steal syndrome and coronary vein varices have also been described in patients with liver cirrhosis, in which portal vein pressure increases [30]. It is also known that total anomalous pulmonary venous drainage [31], chronic pulmonary hypertension [32] and moderate or advanced mitral regurgitation [33] dilate the coronary sinus. Although there are studies reporting coronary vein enlargement, with even case reports of cardiac varicose veins, 
no study has shown that molecules from the veins pass through the myocardial tissue due to increased pressure in the veins, leading to histopathological and immunohistochemical changes. This movement of the molecules due to increased chronic venous pressure produces histopathological lesions, leading to clinical manifestations of well-defined venous insufficiency (e.g., CVI and CCSVI). Further clinical studies are needed to answer the question whether the phenomenon of chronic coronary venous insufficiency is implicated due to similar histopathological findings observed by us. In their anatomical study, Bahar et al. examined the vein distribution of rabbits and observed ampullary dilatation in the segment between an incompetent monocuspid valve, at the junction between the GCV and LCVC, and the bicuspid valve $5 \mathrm{~mm}$ away from this junction. They attributed this ampullary dilation to the pressure of the blood escaping from the LCVC back to the bicuspid valve due to the incompetent monocuspid valve. On the other hand, no dilatation was observed in rabbits with competent monocuspid valves at the GCV-LCVC junction [13]. It is well known that valves are prevalent in human coronary veins [6]. Autopsy studies are needed to answer the question whether chronic coronary venous insufficiency due to venous dilatation and chronic pressure load can be implicated in humans due to incompetent coronary vein valves.

Coronary retroperfusion was performed in humans for the first time in 1956 via the CS to protect and nourish the myocardium during open heart surgery [34]. Gott et al. observed that ecchymosis occurred in the myocardial tissue when the average CS pressure exceeded $40 \mathrm{~mm} \mathrm{Hg}$ while performing coronary retroperfusion during aortic valve surgery; moreover, a systolic pressure of $>80 \mathrm{~mm} \mathrm{Hg}$ led to more widespread damage [35]. In their experimental study, Miyahara et al. occluded the CS of dogs by supplying thrombin to cause thrombus and showed that this caused myocardial infarction [14]. Life-threatening coronary sinus thrombosis after catheter ablation has also been observed in case reports. In this study, the authors examined myocardial injury after coronary sinus thrombosis and suggested that the occlusion of the entire coronary venous system leads primarily to transvascular pressure elevation and secondarily to venous and capillary engorgement, causing direct myocardial damage [15]. Therefore, in order not to occlude the entire venous system, no occlusion was performed in the GCV, where all coronary veins drain, in rabbits. Instead, the PIV, a segment away from the GCV, was ligated.

The present study has some limitations. As it was not within the capabilities of our research laboratory, cardiac veins and cardiac function could not be evaluated using coronary venography or cardiac magnetic resonance imaging. We could not determine coronary venous pressure (in terms of increased $\mathrm{mm} \mathrm{Hg}$ figures) after coronary vein ligation. Although it is reasonable to assume that the pressure in the ligated coronary veins of these rabbits was elevated in this model, this is not certain unless measured because the precise anatomy of the connections and branching of these veins, as well as the presence or absence of valves, may vary across animals as is the case in humans.

In conclusion, this experimental study showed histopathological and immunohistochemical changes in myocardial tissue, similar to those observed in CVI, as a result of increased coronary venous pressure due to coronary vein ligation. Further experimental and clinical studies are needed to understand the clinical implications of these changes occurring in myocardial tissue as a result of increased coronary venous pressure.

\section{Acknowledgments}

This research was supported by the Scientific Research Projects Coordination Unit of Canakkale Onsekiz Mart University as an "Independent Research Project” (Project ID: 2751, Code: THD2018-2751). I would like to thank veterinarian doctor Sait Elmas and the entire staff of Canakkale Onsekiz Mart University experimental research application and research center, for their support during coronary vein ligation; Prof. Dr. Coskun Bakar and Dr. Buse Yuksel for their support in statistical analyses; and Prof. Dr. Armagan Altun for his input and support while finalizing this article.

\section{Conflict of interest}

The authors declare no conflict of interest.

\section{References}

1. Benjamin EJ, Muntner P, Alonso A, et al. Heart disease and stroke statistics - 2019 update: a report from the American Heart Association. Circulation 2019; 139: e1-473.

2. Eberhardt RT, Raffetto JD. Chronic venous insufficiency. Circulation 2014; 130: 333-46.

3. Mansilha A, Sousa J. Pathophysiological mechanisms of chronic venous disease and implications for venoactive drug therapy. Int J Mol Sci 2018; 19: e1669.

4. Ackerman Z, Seidenbaum M, Loewenthal E, Rubinow A. Overload of iron in the skin of patients with varicose ulcers. Possible contributing role of iron accumulation in progression of the disease. Arch Dermatol 1988; 124: 1376-8.

5. Kavala AA, Turkyilmaz S. Autogenously derived regenerative cell therapy for venous leg ulcers. Arch Med Sci Atheroscler Dis 2018; 3: e156-63.

6. Saremi F, Muresian H, Sánchez-Quintana D. Coronary veins: comprehensive $\mathrm{CT}$-anatomic classification and review of variants and clinical implications. Radiographics 2012; 32: e1-32. 
7. Pan-Chih, Huang AH, Dorsey LM. Hemodynamic significance of the coronary vein valves. Ann Thorac Surg 1994; 57: 424-30.

8. Bergan JJ, Pascarella L, Schmid-Schonbein GW. Pathogenesis of primary chronic venous disease: insights from animal models of venous hypertension. J Vasc Surg 2008; 47: 183-92.

9. Hahn TL, Unthank JL, Lalka SG. Increased hindlimb leukocyte concentration in a chronic rodent model of venous hypertension. J Surg Res 1999; 81: 38-41.

10. Zakaria MMA, Mikhael SY, Hussein AKA, et al. Failure of the vascular hypothesis of multiple sclerosis in a rat model of chronic cerebrospinal venous insufficiency. Folia Neuropathol 2017; 55: 49-5.

11. Kehinde BA, Abolhassani F, Yazdekhasti $\mathrm{H}$, et al. The ef fects of unilateral varicose ovarian vein on antioxidant capacity and oocyte quality in rat ovary. Iran J Basic Med Sci 2016; 19: 863-9.

12. Yoldas $A$, Nur IH. The distribution of the cardiac veins in the New Zealand White rabbits (Oryctolagus cuniculus) Iran J Veter Res 2012; 13: 227-33.

13. Bahar S, Tipirdamaz S, Eken E. The distribution of the cardiac veins in Angora rabbits (Oryctolagus cuniculus). Anat Histol Embryol 2007; 36: 250-54.

14. Miyahara K, Satoh F, Sakamoto H. Experimental study of acute coronary sinus thrombosis. Jpn Circ J 1988; 52: 44-52.

15. Yeo KK, Davenport J, Raff G, Laird JR. Life-threatening coronary sinus thrombosis following catheter ablation: case report and review of literature. Cardiovasc Revasc Med 2010; 11: 262e1-5.

16. Leary S, Underwood W, Anthony R, et al. AVMA Guidelines for the Euthanasia of Animals: 2013 Edition. American Veterinary Medical Association, Schaumburg Illinois, USA 2013.

17. Bozkurt S, Arikan DC, Kurutas EB, et al. Selenium has a protective effect on ischemia/reperfusion injury in a rat ovary model: biochemical and histopathologic evaluation. J Pediatr Surg 2012; 47: 1735-41.

18. Takase S, Pascarella L, Bergan JJ, Schmid-Schönbein GW. Hypertension-induced venous valve remodeling. J Vasc Surg 2004; 39: 1329-34.

19. Herrick SE, Sloan P, McGurk M, Freak L, McCollum CN, Ferguson MW. Sequential changes in histologic pattern and extracellular matrix deposition during the healing of chronic venous ulcers. Am J Pathol 1992; 141: 108595.

20. Norgauer J, Hildenbrand T, Idzko M, et al. Elevated expression of extracellular matrix metalloproteinase inducer (CD147) and membrane-type matrix metalloproteinases in venous leg ulcers. Br J Dermatol 2002; 147: 1180-6.

21. Aronow WS. Management of cardiac hemochromatosis. Arch Med Sci 2018; 14: 560-8.

22. Zhabyeyev P, Das SK, Basu R, et al. TIMP3 deficiency exacerbates iron overload-mediated cardiomyopathy and liver disease. Am J Physiol Heart Circ Physiol 2018; 314: 978-90.

23. Ramm GA, Ruddell RG. Hepatotoxicity of iron overload: mechanisms of iron-induced hepatic fibrogenesis. Semin Liver Dis 2005; 25: 433-49.

24. Zamboni P, Galeotti R. The chronic cerebrospinal venous insufficiency syndrome. Phlebology 2010; 25: 269-79.

25. Porto G, De Sousa M. Iron overload and immunity. World J Gastroenterol 2007; 13: 4707-15.

26. Challoumas D, Pericleous A, Dimitrakaki IA, Danelatos C, Dimitrakakis G. Coronary arteriovenous fistulae: a review. Int J Angiol 2014; 23: 1-10.
27. Cohen B, Winer HE, Kronzon I. Echocardiography in persistent left superior vena cava and dilated coronary sinus. Am J Cardiol 1979; 44: 158-63.

28. Snider AR, Dorts TA, Silverman NH. Venous anomalies of the coronary sinus: detection by $\mathrm{M}$-mode, two dimensional and contrast echocardiography. Circulation 1979; 60: 721-25.

29. Moey YYM, Ebin E, Marcu CB. Venous varices of the heart: a case report of spontaneous coronary sinus thrombosis with persistent left superior vena cava. Eur Heart J Case Rep 2018; 2: yty092.

30. Gupta A, Klintmalm GB, Kim PT. Ligating coronary vein varices: an effective treatment of "coronary vein steal" to increase portal flow in liver transplantation. Liver Transpl 2016; 22: 1037-9.

31. Aziz KU, Paul MH, Bharati S, Lev M, Shannon K. Echocardiographic features of total anomalous pulmonary venous drainage into the coronary sinus. Am J Cardiol 1978; 42: 108-13.

32. Mahmud E, Raisinghani A, Keramati S, Auger W, Blanchard DG, DeMaria AN. Dilation of the coronary sinus on echocardiogram: prevalence and significance in patients with chronic pulmonary hypertension. J Am Soc Echocardiogr 2001; 14: 44-9.

33. Lee MS, Shah AP, Dang N, et al. Coronary sinus is dilated and outwardly displaced in patients with mitral regurgitation: quantitative angiographic analysis. Catheter Cardiovasc Interv 2006; 67: 490-4.

34. Lillehei CW, DeWall RA, Gott VL, Varco RL. The direct vision correction of calcific aortic stenosis by means of a pump-oxygenator and retrograde coronary sinus perfusion. Dis Chest 1956; 30: 123-32.

35. Gott VL, Gonzalez JL, Zuhdi MN, Varco RL, Lillehei CW. Retrograde perfusion of the coronary sinus for direct vision aortic surgery. Surg Gynecol Obstet 1957; 104: 319-28. 